ESTUDO SOBRE ADESIGUALDADE DE RENDAE SEUS DETERMINANTES NO BRASIL

Jair Andrade de Araújo

Universidade Federal do Ceará (UFC)

Emerson Marinho

Universidade Federal do Ceará (UFC)

\title{
ESTUDO SOBRE A DESIGUALDADE DE RENDA E SEUS DETERMINANTES NO BRASIL
}

Resumo: A partir de dados em painel dos estados brasileiros no período 1996-2009, o artigo faz uma análise sobre as contribuições de diferentes determinantes para a redução da desigualdade dos rendimentos no Brasil, com base nos dados da Pesquisa Nacional por Amostra de Domicílios (PNAD). Com este objetivo, especifica um modelo econométrico dinâmico que é estimado pelo Método dos Momentos Generalizado-sistema (MMG-sistema). Os resultados estimados do modelo permitem concluir que as transferências de renda do governo não afetaram a dinâmica da desigualdade de renda no período. Em relação aos outros determinantes, aponta que a educação foi quem mais contribuiu para a redução da desigualdade de renda. Por fim, considera que o produto interno bruto e a variável carga tributária do governo contribuíram, também, para a desigualdade de renda no país.

Palavras-chaves: Desigualdade, renda, painel dinâmico.

\section{STUDY ON BRAZILIAN INCOME INEQUALITY AND ITS DETERMINANTS IN BRAZIL}

Abstract: This work is viewed from panel data of Brazilian states towards the 1996-2009's. It also aims to analyze the contribution concerning the different determinants for reducing Brazilian income inequality, which is estimated according to a survey called (PNAD). The idea here is using a dynamic econometric model which is estimated by the Method of Moments of the Generalized-System (MMG-system). The estimated results conclude that governmental income transferring did not have any impact upon the so-called dynamic concerning income inequality to the period sketched. However, if we consider other determinants, point out that education was the mainly contributor to reduce the income inequality. Furthermore, considers that the gross national product and the variable government's tax burden contribute to increase income inequality in Brazil.

Key words: Inequality, income, dynamic panel. 


\section{INTRODUÇÃO}

No Brasil, a desigualdade de renda cresceu na década de setenta e se manteve elevada até a metade da década de noventa. Essa situação começou a se alterar depois da implantação do Plano Real, quando os índices de desigualdade começaram a apresentar redução. O grande foco dos estudos de desigualdade no país tem sido sobre a existência histórica de profundos desníveis sociais nas condições de vida, e principalmente de renda entre os residentes das regiões brasileiras.

É nesse contexto que surge a principal motivação e objetivo deste estudo: propõe-se realizar análise das contribuições de diferentes determinantes para a redução da desigualdade dos rendimentos no País, entre 1996 a 2009. A principal interrogação que se coloca em torno dessa questão diz respeito sobre quais são os determinantes que contribuíram para a queda da desigualdade no Brasil. Será que os programas de transferência de renda do governo federal ajudaram a diminuir a desigualdade? Ou será que alguns outros fatores determinaram de forma mais significativa a queda da desigualdade de renda no país?

O objetivo deste artigo é dar elementos de respostas a estas perguntas. Para isto, serão feitas estimações que expliquem a desigualdade de renda no Brasil. Espera-se que estas informações sirvam de subsídio para que se formulem decisões sobre os fatores que contribuíram para queda do índice de Gini no país durante 1996 a 2009.

Isto posto, para alcançar os objetivos acima descritos, será estimado um modelo dinâmico para dados em painel, desenvolvidos por Arellano e Bond (1991), Arellano e Bover (1995) e Blundel e Bond (1998). Nesse modelo, a variável dependente será: o coeficiente de Gini e as variáveis explicativas serão: o coeficiente de Gini defasados em um período; produto interno bruto (PIB); anos de estudo; transferência de renda do governo; rendimentos do trabalho e impostos arrecadados pelo governo. Os dados utilizados nessa estimação foram obtidos nas Pesquisa Nacional por Amostra de Domicílios (PNAD), nas bases de dados do Ministério da Fazenda e do IPEADATA, no período de 1996 a 2009.

O artigo é composto por seis seções, incluindo esta introdução. A segunda seção faz um breve histórico da desigualdade brasileira, além de mostrar a sua relação com as transferências de renda do governo e outros determinantes. A seção 3 define e discute a base de dados. A quarta seção apresenta o modelo econométrico e os métodos de estimação empregados. A quinta seção analisa os resultados obtidos da estimação do modelo econométrico. Por último, as conclusões são comentadas na seção 6 .

\section{DESIGUALDADE E SEUS DETERMINANTES}

Verifica-se no Brasil, em anos recentes, particularmente a partir de 2001, o declínio da desigualdade. Segundo Barros, Franco e Mendonça (2007) entre 2001 e 2005 o grau de desigualdade de renda no Brasil declinou de forma acentuada e contínua, atingindo, em 2005 o nível mais baixo dos últimos anos. O coeficiente de Gini diminuiu quase $5 \%$ e a razão entre a renda dos $20 \%$ mais ricos e a dos $20 \%$ mais pobres reduziu-se em $20 \%$. Essa redução contribuiu para diminuir substancialmente a pobreza e melhorar as condições de vida da população mais pobre, mesmo em um período de relativa estagnação da renda per capita.

A Tabela 1, a seguir, mostra a evolução do coeficiente de Gini, que mede o grau de desigualdade existente na distribuição de indivíduos segundo a renda familiar per capita, para a economia brasileira e para as macrorregiões no período de 1996 a 2009. Pode-se ver que o coeficiente de Gini para o Brasil caiu de 0,602 para 0,543 no período analisado, 0 que significou uma redução de 0,059 pontos, ou de $9,80 \%$. É interessante observar que a queda na desigualdade não foi ininterrupta, pois, de 1999 a 2001, ocorreu uma elevação desse coeficiente.

A mesma Tabela mostra que as regiões Sul, Nordeste, Norte e Centro-Oeste, nesta ordem, apresentaram as maiores reduções no coeficiente de Gini. Em 1996, a região Nordeste apresentava a maior desigualdade de renda entre as regiões, seguida pela região Centro-Oeste. Em 2009, essa última região apresentou a pior distribuição de renda entre as regiões, deixando o segundo lugar para o Nordeste.

Os dados da Tabela 1 mostram que nem todas as regiões apresentaram queda ininterrupta na desigualdade de renda medida pelo Gini no período analisado. A única região que apresentou sucessivas reduções na desigualdade foi o Nordeste. A região Sudeste, apesar da diminuição do Gini de 1996 para 2009, apresentou uma elevação nos anos de 1997 , 1998 e 2001. As regiões Centro-Oeste, Sul e Norte também não apresentaram quedas ininterruptas da desigualdade.

Os resultados mostrados evidenciam que a desigualdade declinou no Brasil nos últimos anos. Depois de verificada essa redução, alguns trabalhos na literatura nacional procuraram identificar quais os principais determinantes desta queda. As subseções a seguir irão abordar de forma mais detalhada esses determinantes.

\subsection{Relação entre Desigualdade e os Programas de Transferências de Renda}

O objetivo dessa subseção é relatar as evidências na literatura sobre os impactos dos programas de transferência de renda na redução das desigualdades e pobreza. A princípio pode-se dizer 
Tabela 1 - Coeficiente de Gini: Brasil e Regiões - 1996 e 2009

\begin{tabular}{c|cccccc}
\hline Ano & Brasil & Nordeste & Sudeste & Sul & Centro-Oeste & Norte \\
\hline 1996 & 0.602 & 0.620 & 0.563 & 0.561 & 0.601 & 0.580 \\
1997 & 0.602 & 0.617 & 0.565 & 0.555 & 0.599 & 0.587 \\
1998 & 0.600 & 0.610 & 0.566 & 0.557 & 0.603 & 0.583 \\
1999 & 0.594 & 0.605 & 0.559 & 0.562 & 0.593 & 0.565 \\
2001 & 0.596 & 0.600 & 0.568 & 0.548 & 0.598 & 0.565 \\
2002 & 0.589 & 0.595 & 0.563 & 0.529 & 0.595 & 0.564 \\
2003 & 0.583 & 0.585 & 0.557 & 0.531 & 0.581 & 0.542 \\
2004 & 0.572 & 0.583 & 0.542 & 0.522 & 0.573 & 0.539 \\
2005 & 0.569 & 0.571 & 0.543 & 0.515 & 0.577 & 0.529 \\
2006 & 0.563 & 0.573 & 0.538 & 0.506 & 0.563 & 0.521 \\
2007 & 0.556 & 0.566 & 0.524 & 0.505 & 0.574 & 0.533 \\
2008 & 0.548 & 0.558 & 0.518 & 0.495 & 0.568 & 0.509 \\
2009 & 0.543 & 0.558 & 0.511 & 0.491 & 0.560 & 0.523 \\
\hline Diferença & -0.059 & -0.062 & -0.052 & -0.070 & -0.041 & -0.057
\end{tabular}

Fonte: Elaborado pelo autor, conforme dados da PNADs.

que não existe consenso entre os pesquisadores sobre esses impactos.

Analisando esses programas de transferência de renda nos Estados Unidos, Enders e Hoover (2003) concluem que eles não têm efeito significativo sobre a pobreza. Ressaltam que na literatura internacional não são claros os impactos das transferências de renda às famílias pobres sobre a redução da pobreza e desigualdades.

O governo brasileiro adotou nos últimos anos políticas de transferência de renda com o objetivo de combater a pobreza e desigualdades. O Brasil tinha quatro programas de transferência de renda até outubro de 2003 , quando ocorreu a unificação dos programas de transferência de renda mensal a partir dos programas existentes, criando o programa Bolsa Família, inspirado pelo programa de renda mínima vinculado à educação, o Bolsa Escola.

DeacordocomoMinistério do Desenvolvimento Social e Combate á Fome (MDS) o Bolsa Família beneficiou, mensalmente, 10.830 .580 famílias no Brasil, em 2008. Esse programa beneficia famílias cuja renda familiar per capita seja inferior a $R \$$ 60,00 mensais e famílias de gestantes, crianças e adolescentes de até 15 anos cuja renda per capita seja inferior a $\mathrm{R} \$ 120$ (BRASIL, [2008]).

No entanto, vários trabalhos mostram que existem muitas controvérsias quanto à eficácia dessa política. Alguns autores afirmam que as transferências governamentais desestimulam os indivíduos pobres a procurar emprego e os tornam dependentes das transferências contínuas do governo. Por outro lado, outros estudos apontam para a eficácia desses programas.

Segundo Rocha (2006), embora esses programas apresentem problemas de focalização, ocorreu um aumento de cobertura no Brasil contribuindo para redução da pobreza e da indigência, o que corrobora os resultados de Kakwani, Neri e Son (2006). Esses últimos afirmam que esses programas no Brasil são responsáveis por uma fração pequena na queda da desigualdade de renda nos últimos anos.

Por outro lado, Marinho, Linhares e Câmpelo (2007) discordam desses resultados. Através de um painel dinâmico nos anos de 1996 a 2006, com dados das PNADs, concluíram que essa política não apresentou impacto significativo sobre a pobreza no Brasil, mas que a queda da desigualdade teve forte impacto na redução da mesma.

$\mathrm{Em}$ assim sendo, procura-se verificar a influência desses programas de transferência de renda sobre a desigualdade de renda no Brasil, formalmente, por meio do modelo econométrico a ser especificado na seção 4.

\subsection{Relação entre desigualdade e crescimento econômico}

Crescimento econômico e desigualdade têm sido estudados por diversos autores, mas apesar disto não existe consenso no sinal desta relação. O principal objetivo desta subseção tem origem na literatura econômica que trata da forma como a desigualdade de renda afeta o crescimento econômico.

Alguns autores sugerem que a desigualdade pode estimular o crescimento; outros afirmam que a desigualdade poderia arrefecer o crescimento, enquanto um terceiro grupo afirma que este último não tem impacto sobre a desigualdade.

A relação entre a distribuição de renda e o processo de crescimento econômico já foi analisado por diversos autores. O vínculo entre esses dois fenômenos foi relatado de maneira clara por Kuznets (1955). 
Com base na evidência de dados de séries de tempo, Kuznets (1955) apresentou a relação de $U$ invertido entre a renda e desigualdade, onde afirma que no início do processo de crescimento, ocorre aumento da desigualdade. Após o nível de renda per capita das economias ultrapassarem certo nível, a desigualdade começa a diminuir. Esse padrão ficou conhecido na literatura econômica como a curva de Kuznets. Este autor tinha em mente sociedades que transitavam das atividades rurais para as industriais. Inicialmente, alguns indivíduos se beneficiariam mais do que outros dos frutos da industrialização, até o momento em que ela fosse predominante na sociedade e a maioria pudesse se beneficiar dos seus resultados.

Existem diversos trabalhos que mostram evidências empíricas da curva de Kuznets, inclusive para o Brasil. Por exemplo, Barreto, Jorge Neto e Tebaldi (2001), utilizando o método dos momentos generalizados, encontram que a concentração de renda gerou externalidades negativas para o Nordeste e prejudicou, assim, o crescimento do produto per capita.

Ao contrário, Aghion e Bolton (1992) afirmam que a desigualdade de renda afeta o crescimento devido às imperfeições no mercado de capitais que limita o acesso dos agentes mais pobres ao financiamento de atividades lucrativas. Assim, os indivíduos pobres ficam incapacitados de investirem em capital humano devido à restrição ao crédito, inibindo o crescimento.

Verifica-se que nos diversos estudos acima citados existe uma incessante busca em identificar as relações entre desigualdade e crescimento. A discussão sobre essa questão continua em aberto na literatura da área.

\subsection{Relação entre desigualdade e renda do trabalho}

Neste item, busca-se referenciar a relação entre desigualdade e renda do trabalho através de artigos na literatura nacional e internacional. Normalmente, esses artigos utilizam de modo sistemático a renda familiar do trabalho, isto é, a remuneração derivada do trabalho de todos os membros da família. Para o Brasil a maioria desses estudos demonstra que a maior parcela na queda da desigualdade de renda nos últimos anos se deve a mudanças na distribuição dos rendimentos do trabalho.

Por exemplo, Hoffmann (2006), utilizando a metodologia de decomposição da variação para o índice de Gini no Brasil, estimou que no período 2002-2005 a variação desse índice foi de $-0,0185$, verificando que $69 \%$ dessa variação está associada ao rendimento de todos os trabalhos e $31,4 \%$ ao crescimento das rendas de transferências do governo.
$\mathrm{Na}$ mesma perspectiva, Azevedo e Foguel (2007), por meio da decomposição da desigualdade dos rendimentos do trabalho no Brasil, para o período compreendido entre 1995 e 2005, verificaram que quase todas as medidas de desigualdade mostraram uma redução da concentração dos rendimentos do trabalho. Ressaltam que 0 efeito preço desempenhou o papel mais importante para explicar a queda recente na desigualdade de rendimentos do trabalho.

\subsection{Relação entre Desigualdade e Tributação}

De forma geral os trabalhos na literatura nacional sobre esse tema confirmam a hipótese de que a carga tributária no Brasil representa um fator que atua no sentido de manter, quando não de aprofundar, os níveis de desigualdade social do país.

Por exemplo, Viana e outros (2001), estudando as relações entre desigualdade e arrecadação tributária no Brasil não encontram nenhuma relação de causalidade e nem afirmam categoricamente que a tributação se constitui no principal fator para explicar a concentração de renda no país. No entanto, são enfáticos ao afirmarem que o sistema tributário brasileiro da forma como está estruturado é um dos fatores a contribuir para a manutenção do quadro de distribuição desigual da renda, e, portanto, dos níveis de pobreza e de indigência.

Nessa mesma perspectiva, Rocha (2006) mostra que o nível ótimo da carga tributária no Brasil é em torno de $25 \%$ do PIB, a partir do qual a carga tributária passa a deprimir o crescimento de longo prazo prejudicando a distribuição de renda.

Recentemente, Conde (2008) afirma que o sistema tributário mais justo deve ser progressivo, e não regressivo como é atualmente. Salienta que os impostos indiretos (aqueles embutidos nos preços dos produtos e serviços) são um dos principais potencializadores da desigualdade brasileira. Os pobres pagam proporcionalmente três vezes mais Imposto sobre Circulação de Mercadoria e Serviços (ICMS) que os ricos. Enquanto estes últimos desembolsam em média $5,7 \%$ de ICMS, os pobres pagam $16 \%$ do mesmo imposto.

\subsection{Relação entre Desigualdade e Educação}

Em vários trabalhos, o investimento em capital humano tem-se mostrado cada vez mais importante como um dos fatores determinantes da redução da desigualdade. Os países desenvolvidos já tinham percebido há bastante tempo que esta variável era relevante para o aumento da produtividade do trabalhador. Isso implicava em aumento de rendimento dos trabalhadores, ajudando a reduzir as desigualdades existentes.

A expressão capital humano aparece no trabalho de Schultz (1960) estudando as situações 
das nações subdesenvolvidas. O autor afirma que aumento no bem-estar dos pobres não dependia da terra, dos equipamentos ou da energia, mas sim do conhecimento. Portanto, a educação tem como função precípua desenvolver habilidades e conhecimentos objetivando o aumento da produtividade e maiores ganhos de habilidades cognitivas. Afinal, quanto maior for o grau de produtividade maior será a cota de renda que a pessoa receberá e melhor será sua posição social contribuindo para reduzir as desigualdades.

De forma geral, os estudos sobre a relação entre desigualdade de renda e educação destacam que no Brasil a desigualdade é, em grande parte, resultado da péssima distribuição educacional existente, tanto em termos pessoais como entre grupos de indivíduos com características similares. No país os ricos têm acesso à educação mais qualificada que os pobres, contribuindo, assim, para uma sociedade mais desigual.

Segundo Enrenberg e Smith (2000), o aumento no nível de educação resulta em acréscimos de produtividade, que por sua vez, eleva o nível de salário real. Dessa forma, as regiões que possuem maior estoque de capital humano tendem a apresentar um salário médio superior às das demais localidades. Além da elevação do salário, a concentração de conhecimentos gera externalidades positivas para a região. O padrão de crescimento desta região se torna mais dinâmico induzindo a entrada de novos investimentos e propagação de novos conhecimentos e habilidades.

\section{BASE DE DADOS}

A maioria das variáveis utilizadas ou foi construída ou diretamente extraída das PNADs para o período de 1996 a 2009¹. Os dados se referem aos estados brasileiros, considerando apenas as regiões Nordeste, Centro-Oeste, Sul e Sudeste ${ }^{2}$.

As PNADs até 2004 não identificam os beneficiários dos programas de transferências de renda do governo federal destinados às famílias pobres dentro dos domicílios, nem faz a distinção entre rendas recebidas desses programas e rendimentos de aplicações financeiras. Assim sendo, para calcular o valor das transferências construiu-se um filtro para esta rubrica, selecionando as pessoas cuja renda per capita familiar fosse igual ou menor do que meio salário mínimo vigente no ano. Supõese que esse resíduo represente a renda advinda de todas as transferências de renda do governo, pois é de se esperar que os indivíduos selecionados por meio desse filtro não devem ter rendimentos de aplicações financeiras. A correlação estimada entre essa variável e a desigualdade irá responder se as transferências impactam ou não a desigualdade de renda.

A variável renda familiar per capita é obtida dividindo-se o rendimento total da família pelo número de componentes. É essa a variável que é utilizada para construir o coeficiente de Gini para cada estado em cada ano da amostra. Para se calcular o coeficiente de Gini, ordena-se de forma crescente o conjunto de renda familiar per capita para se obter a curva de Lorenz. Essa curva relaciona em cada percentual a fração acumulada da população á fração acumulada da renda, e, por meio dela, calcula-se o índice para cada unidade da federação.

A variável renda do trabalho corresponde à renda de todos os trabalhos das pessoas ocupadas. A análise apresentada na subseção 2.3 concluiu que o aumento da renda do trabalho contribui para a queda da desigualdade de renda. Portanto, o sinal do parâmetro estimado dessa variável no modelo econométrico especificado na seção seguinte deve ser negativo.

Para construir uma proxy para a carga tributária, somaram-se todos os impostos e contribuições federais administrados pela Receita Federal do Brasil mais o ICMS extraído da base de dados do Ministério da Fazenda e do IPEADATA respectivamente. De acordo com o que foi discutido na subseção 2.4 , espera-se que essa variável contribua para elevar a desigualdade de renda. Em assim sendo, o sinal esperado do coeficiente estimado dessa variável no modelo econométrico da seção seguinte deve ser positivo.

A variável educação utilizada é a média de anos de estudo dos residentes dos estados brasileiros. Os vários artigos apresentados na subseção 2.5 mostraram que a educação, em geral, contribui para diminuir a desigualdade de renda. Dessa forma, a correlação entre a desigualdade e a educação deve ser negativa.

Além disso, foi usada a variável Produto Interno Bruto dos estados (PIB) extraídos do IPEADATA a preços constantes do ano base de 2009. O sinal estimado do parâmetro dessa variável no modelo deve ser negativo. Pois, quanto mais rico seja o estado, maiores as externalidades positivas sobre os que nele residem. Se isso é verdade, o efeito pode vir a diminuir a desigualdade.

No mais, ressalta que todas as variáveis monetárias foram atualizadas para valores reais de 2009, utilizando o Índice Nacional de Preços ao Consumidor (INPC), tendo como base o ano de 2009.

\section{MODELO ECONOMÉTRICO}

O modelo supõe que o quadro de desigualdade de renda corrente tende a se perpetuar e/ou influenciar o desempenho da desigualdade no futuro. A relação entre a desigualdade de renda e seus determinantes é investigada por meio do seguinte modelo de regressão para dados em painel:

$\ln \left[\right.$ Gini $\left._{i t}\right]=\beta_{0}+\beta_{1} \ln \left[{\left.\text { Gini } i_{i-1}\right]}\right]+\beta_{2} \ln \left[P I B_{i t}\right]+\beta_{3} \ln \left[\right.$ Ame $\left._{i t}\right]+\beta_{4} \ln \left[\right.$ Transf $_{i t}$ 
onde, a variável coeficiente de Gini $_{\text {itt }}$ é a medida de desigualdade de renda; $P I B_{i t}$ é o Produto Interno Bruto; $A m e_{i t}$ são os anos médios de estudo

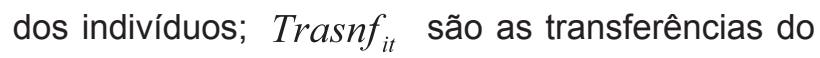
governo federal; Re $n t_{i t}$ é a renda do trabalho dos indivíduos; Im post $_{i t}$ são os impostos e contribuições federais mais o ICMS; $\eta_{i}$ são os efeitos aleatórios não observáveis dos indivíduos e $\varepsilon_{i t}$ representa os distúrbios aleatórios. As variáveis do modelo (1) são definidas em logaritmo natural em que o subscrito $i$ representa o estado e $t$ o período de tempo.

$$
\text { Conforme Ahn e Schmidt (1995), }
$$
esse modelo possui as seguintes hipóteses: $E\left[\eta_{i}\right]=E\left[\varepsilon_{i}\right]=E\left[\eta_{i} \varepsilon_{i}\right]=0$ e $E\left[\varepsilon_{i t} \varepsilon_{\dot{b}}\right]=0$ para $i=1,2, \ldots . ., N$ e $\forall t \neq s$. Há também a hipótese padrão relativa às condições iniciais inini $_{\dot{t}-1}$ : $E\left[\operatorname{Gini}_{\dot{t}-1} \varepsilon_{i}\right]=0$ para $i=1,2, \ldots, N$ e $t=1,2, \ldots ., T$. O trabalho de Arellano e Bond (1991) destaca que ocorrem dois problemas econométricos ao estimar o modelo (1) por meio de técnicas de estimação tradicionais.

Primeiro, devido à presença dos efeitos não observáveis dos indivíduos, ${ }^{\text {Ginitit-1 }_{i}}$, juntamente com a variável dependente defasada, Gini $i_{i t-1}$, no lado direito da equação. Nesse caso, omitir os efeitos fixos individuais no modelo dinâmico em painel torna os estimadores de mínimos quadrados ordinários (MQO) enviesados e inconsistentes. Entretanto, o estimador WITHIN GROUPS, que corrige para

presença de efeitos fixos, gera uma estimativa de $\beta_{1}$ enviesada para baixo em painéis com a dimensão temporal pequena.

Segundo, devido à provável endogeneidade das variáveis explicativas. Nesse caso, endogeneidade no lado direito da equação (1) deve ser tratada para evitar um possível viés gerado por problema de simultaneidade.

Uma maneira de solucionar esses problemas, Arellano e Bond (1991) propõem o estimador do método dos momentos generalizado-diferenciado (MMG-diferenciado). Tal método consiste na eliminação dos efeitos fixos através da primeira diferença da equação (1) da seguinte forma:

$\Delta \ln \left[\operatorname{Gin}_{i t}\right]=\beta_{1} \Delta \ln \left[\operatorname{Gini}_{, i t-1}\right]+\beta_{2} \Delta \ln \left[P I B_{i t}\right]+\beta_{3} \Delta \ln \left[\right.$ Ame $\left._{i t}\right]+\beta_{4} \Delta \ln \left[\operatorname{Trans}_{t i}\right]+\beta_{5} \Delta \ln \left[\operatorname{Rent}_{i t}\right]+$

onde, para qualquer variável $\ln y_{i t}$, $\Delta \ln y_{i t}=\ln y_{i t}-\ln y_{i t-1}$. Note que naequação (2), $\Delta \ln \operatorname{Gini}_{i t-1}$ e $\Delta \ln \varepsilon_{i t}$ são correlacionados e, assim sendo, estimadores de MQO para seus coeficientes serão enviesados e inconsistentes. Portanto, faz-se necessário utilizar variáveis instrumentais para .

As hipóteses adotadas na equação (1) implicam que as condições de momentos $E\left[\Delta \mathrm{h} \mathrm{Gini}_{, i t-s} \Delta \mathrm{h} \varepsilon_{t}\right]=0$, para $t=3,4, \ldots . T$ e $s \geq 2$, são válidas. Baseados nesses momentos, Arellano e Bond (1991) sugerem empregar h Gini $_{\text {tit-s }}$, para $t=3,4, \ldots . T$ e $s \geq 2$, como instrumentos para equação (2).

As demais variáveis explicativas podem ser classificadas como: (a) estritamente exógena, se não é correlacionada com os termos de erro passados, presente e futuros; (b) fracamente exógena, se é correlacionada apenas com valores passados do termo de erro e (c) endógena, se é correlacionada com os termos de erro passados, presente e futuros. No segundo caso, os valores da variável defasada em um ou mais períodos são instrumentos válidos na estimação da equação (2) e no último caso os valores defasados em dois ou mais períodos são instrumentos válidos na estimação dessa equação.

Conforme Arellano e Bover (1995), Blundell e Bond (1998), esses instrumentos são fracos quando as variáveis dependentes e explicativas apresentam forte persistência e/ou a variância relativa dos efeitos fixos aumenta. Isso produz um estimador MMG-diferenciado não consistente e enviesado para painéis com $\mathrm{T}$ pequeno.

Assim sendo, Arellano e Bover (1995), Blundell e Bond (1998) propõem um sistema que combina o conjunto de equações em diferença, equação (2), com o conjunto de equações em nível, equação (1) para reduzir esse problema de viés. Esse sistema é denominado método dos momentos generalizadosistema (MMG-sistema). Daí surge o método dos Momentos Generalizado-sistema (MMG-sistema). Para as equações em diferenças, o conjunto de instrumentos é o mesmo descrito acima. Para regressão em nível, os instrumentos apropriados são as diferenças defasadas das respectivas variáveis. Por exemplo, assumindo que as diferenças das variáveis explicativas não são correlacionadas com os efeitos fixos individuais (para $t=3,4, \ldots . T$ ) e

$$
E\left[\Delta \mathrm{h} \operatorname{Gini}_{k, i 2} v_{i}\right]=0 \text {, para } i=1,2,3, \ldots, N \text {, então as }
$$

variáveis explicativas em diferenças e $\Delta \mathrm{h} \operatorname{Gini}_{k, t-1}$, caso elas sejam exógenas ou fracamente exógenas, são instrumentos válidos para equação em nível. O mesmo se dá se elas são endógenas, mas com os instrumentos sendo as variáveis explicativas em diferenças defasadas de um período e $\Delta \mathrm{h} \operatorname{Gini}_{k, t-1}$

As estimativas do MMG-sistema apresentadas na próxima seção resultam da avaliação com estimador corrigido pelo método de Windmeijer 
(2005) para evitar que o respectivo estimador das variâncias subestime as verdadeiras variâncias em amostra finita. O estimador utilizado foi proposto por Arellano e Bond (1991) em dois passos. Na primeira etapa, supõe-se que os termos de erro são independentes e homocedásticos nos estados e ao longo do tempo. No segundo estágio, os resíduos obtidos na primeira etapa são utilizados para construir uma estimativa consistente da matriz de variância-covariância, relaxando assim as hipóteses de independência e homocedasticidade. O estimador do segundo estágio é assintoticamente mais eficiente em relação ao estimador da primeira etapa.

Por fim, como forma de testar a robustez e consistência do modelo, Arellano e Bond (1991) sugerem dois tipos de testes. O teste de Sargan, utilizado com o objetivo de verificar a validade dos instrumentos. A falha em rejeitar a hipótese nula indicará que os instrumentos são robustos. Além disso, como se supõe, inicialmente, que o erro

$\varepsilon_{\text {it }}$ não seja autocorrelacionado, é feito um teste de correlação serial de primeira ordem e outro de segunda ordem sobre os resíduos em primeira diferença, $\Delta \varepsilon_{i t}$. Espera-se que esses erros sejam correlacionados em primeira ordem e não autocorrelacionados em segunda ordem.

\section{RESULTADOS DO MODELO ECONOMÉTRICO}

Nesta seção apresentam-se os resultados da estimação do modelo (1) que relaciona a desigualdade de renda medida pelo índice de Gini e seus determinantes.

Além dos resultados das estimações obtidas por MQO, WITHIN GROUPS, apresentam-se também as estimações por meio do método MMGsistema. Como discutido anteriormente, esse método resulta de uma extensão do estimador original de Arellano e Bond (1991), proposta em Arellano e Bover (1995) e desenvolvida em Blundell e Bond (1998) (Tabela 2).

$\mathrm{Na}$ coluna [a], o modelo foi estimado por MQO, obtido para um total de 260 observações, englobando todos os Estados Brasileiros. Com

exceção das transferências do governo ( $G i n i_{t, t-1}$ ), que se revela insignificante, as demais variáveis apresentam sinais significativos conforme esperado. Contudo, esses estimadores são enviesados e inconsistentes como já discutido anteriormente. De fato, observa-se que seus coeficientes são maiores do que os valores estimados na coluna [b] para a

variável defasada Gini $_{t, t-1}$ por WITHIN GROUPS.

A forma de analisar o desempenho dos estimadores MMG-sistema baseia-se na comparação das estimativas para o coeficiente da variável defasada $G i n i_{t, t-1}$, obtidas por meio desse método, e aquelas encontradas por métodos alternativos cujas propriedades são conhecidas em modelos de painel dinâmico.

Como discutido na metodologia, as estimativas de MQO e WITHIN GROUPS são enviesadas para cima e para baixo, respectivamente, fornecendo aproximadamente limite superior e inferior para

o coeficiente $\beta_{1}$. O valor encontrado por MMGsistema para essa variável na coluna [c] na Tabela 2 indica que essa condição foi atendida. Isso significa, portanto, que o viés causado pela presença de variáveis endógenas no lado direito da regressão e efeitos fixos não observáveis foram corrigidos.

Os testes efetuados no modelo MMG-sistema revelam que as propriedades estatísticas do modelo são aceitáveis. Os testes de Hansen e Sargan que testam, respectivamente, se os instrumentos utilizados e os instrumentos adicionais requeridos pelo MMG-sistema são válidos, são satisfatórios.

Por último, incluem-se ainda os testes estatísticos de Arellano e Bond (1991) para avaliar a existência de autocorrelação de primeira e segunda ordem. Note-se que a ausência de autocorrelação de segunda ordem é essencial para a consistência do estimador MMG-sistema. O teste confirma a não rejeição de autocorrelação de primeira ordem, embora se rejeite a hipótese de autocorrelação de segunda ordem. Assim sendo, entre os diversos modelos estimados, optou-se por analisar o modelo apresentado na coluna [c] da Tabela 2.

Verifica-se que no modelo MMG-sistema, o coeficiente da variável dependente defasada apresentou um valor altamente significativo relativamente mais baixo do que o estimador MQO, confirmando a expectativa da persistência da desigualdade de renda no Brasil. Entretanto, a magnitude e o sinal do parâmetro estimado dessa variável indicam que o crescimento da desigualdade de renda é não explosivo. De fato, as informações apresentadas na seção 2 mostraram que, apensar do declínio, a desigualdade de renda no Brasil é persistente durante o período analisado.

Nesse estudo, a variável transferência de renda

(Trans $f_{\text {it }}$ ) apresentou coeficiente insignificante, ou seja, esses programas parecem não estar contribuindo para diminuição da desigualdade de renda no Brasil. Esse resultado está de acordo com a ideia de que as transferências de renda do governo não modificam as distribuições de renda, como já foi discutido na segunda seção.

De certa forma, esse resultado corrobora com o trabalho de Marinho, Linhares e Câmpelo (2007). Segundo os autores, os programas de transferências de renda no Brasil não contribuem para reduzir a pobreza, e os indivíduos que recebem renda desses programas não possuem incentivos para buscar outros meios de obtê-la, tornando-os, assim, dependentes dos programas. Assim, os indivíduos poderiam estar condicionados a permanecer na 
Tabela 2 - Resultados dos Modelos de Regressão para InGini

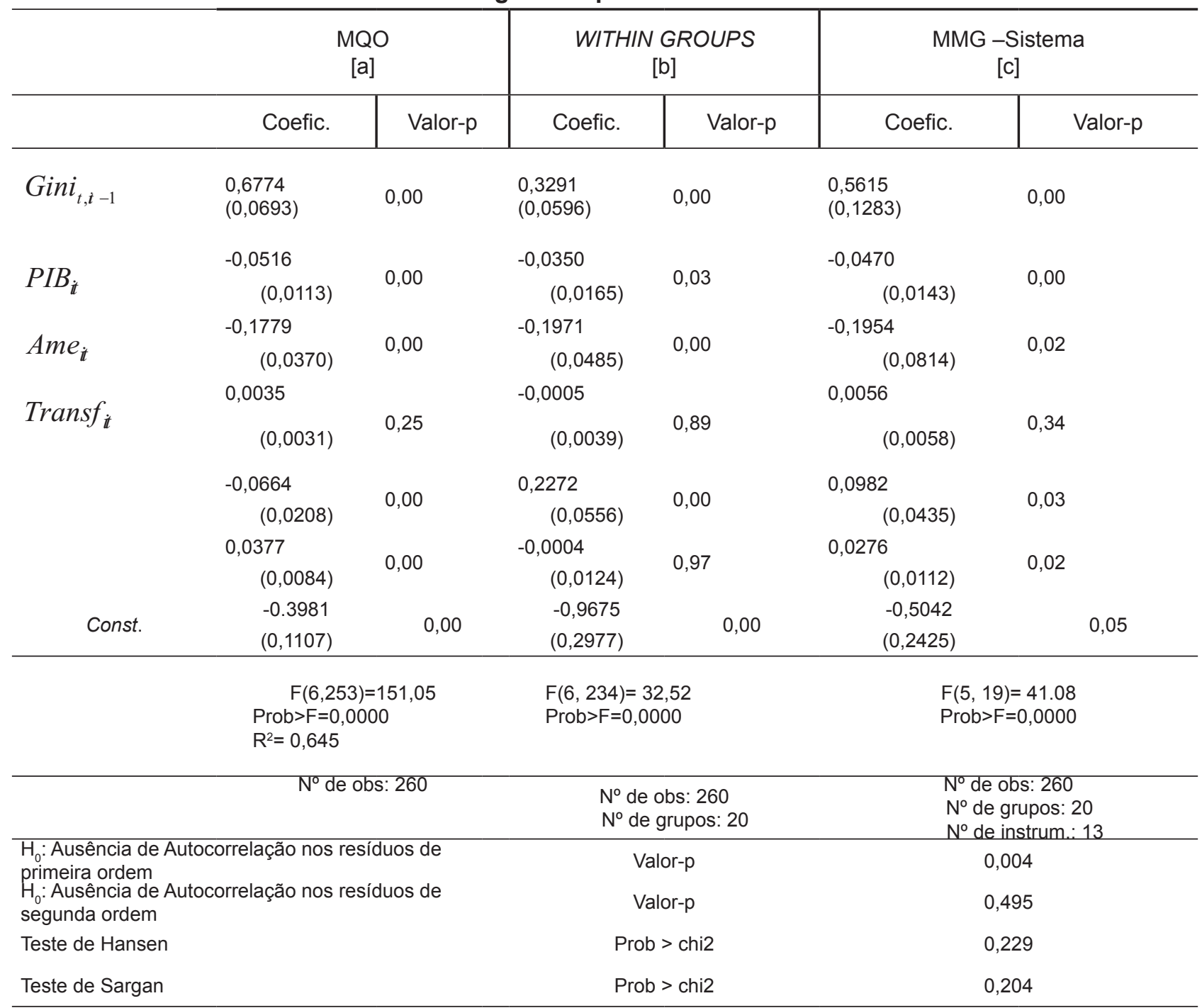

Fonte: Elaborado pelo autor.

Nota: (i) Os valores em parênteses são os desvios padrões corrigidos pelo método de Windmeijer (2005); (ii) Os valores para o teste de Hansen são os valores-p para a hipótese nula de que os instrumentos são válidos e (iii) Os valores para o teste de Sargan são os valores-p para validade dos instrumentos adicionais requeridos pelo método MMG-sistema. (iii) Utilizaram-se como instrumentos no MMG-Sistema as variáveis explicativas em diferenças defasadas e $\Delta \mathbf{h} \operatorname{Gini}_{t, t-1}$ e $\Delta \mathbf{h} P I B_{i t}$ defasadas de um período.

pobreza para continuar recebendo esses benefícios. Outra explicação apresentada é direcionada à gestão desses programas que provavelmente não estaria sendo destinada a quem de fato precisa. De todo modo, os resultados mostram que esses programas não alcançam os objetivos propostos.

Verifica-se a existência de uma relação negativa entre desigualdade de renda e anos médios de estudos, significativa em todos os modelos estimados, colunas [a], [b] e [c]. Por exemplo, na coluna [c] o coeficiente foi de $-0,1954$, ou seja, uma elevação de $1 \%$ no capital humano corresponderia a uma redução de aproximadamente $0,19 \%$ na desigualdade de renda.

Desta forma, concorda-se com diversos autores tais como Schultz (1961), Enreberg e Smith (2000) que afirmam que o aumento no número de estudos dos indivíduos desenvolve habilidades e conhecimentos, elevando produtividade. Isto permite às pessoas adquirir salários mais elevados, diminuindo as desigualdades de renda. Assim, políticas públicas educacionais de qualidade parecem apresentar impactos significativos na redução da desigualdade.

Sugere-se, portanto, políticas públicas que proporcionem incrementos de capital humano, que seriam efetivas para redução das desigualdades.

Dentre os outros determinantes considerados, que afetam as desigualdades de renda, O PIB contribui significativamente para a sua redução. As elasticidades estimadas para o efeito do PIB na redução da desigualdade foi de $0,047 \%$ (valores na coluna [c] da Tabela 2). Essa relação permite concluir que o crescimento econômico contribuiu na redução da desigualdade.

O modelo MMG-sistema mostra a relação positiva e significativa entre renda do trabalho $\left(\operatorname{Re} n t_{i t}\right)$ e desigualdade de renda, apresentando 
elasticidade de 0,0981\%. Desta forma, a renda do trabalho estaria contribuindo para elevação das desigualdades. De fato, ressalta-se que esse resultado não apresentou sinal esperado economicamente e não concorda com autores que afirmaram que a remuneração do trabalho contribuiu para queda da desigualdade em renda no Brasil nos últimos anos.

No presente estudo, identificou-se uma relação positiva, e estatisticamente significativa, entre a arrecadação de impostos e desigualdade de renda com elasticidade de $0,027 \%$ na coluna [c]. Este resultado corrobora os argumentos de Silveira (2004), de que a tributação eleva o nível de desigualdade, mostrando-se altamente perversa aos mais pobres, diminuindo a já precária participação dos pobres na renda global. Tal como no estudo de Rocha (2006), que obtém um nível ótimo de carga tributária do Brasil em torno de $25 \%$ do PIB, mostra que a partir deste valor os impostos passam a deprimir o crescimento, prejudicando a distribuição de renda. Como se sabe, a carga tributária brasileira é superior a esse percentual.

\section{CONCLUSÃO}

Este artigo analisou a desigualdade de renda no Brasil, através da metodologia de dados em painel, utilizando o método dos momentos generalizados em sistema (MMG-Sistema). Dessa forma, foi possível amenizar problemas econométricos, que afetam a maioria dos trabalhos nesta área, como endogeneidade das variáveis explicativas.

Conclui-se, em primeiro lugar, que as transferências de renda não afetam a dinâmica da desigualdade de renda no período, não corroborando, assim, a hipótese daqueles que afirmam que esses programas contribuem para reduzir as desigualdades.

De acordo com os resultados no modelo econométrico, pode-se ver que a variável educação é o principal determinante da desigualdade de renda. Observou-se que para cada aumento de $1 \%$ nos anos médios de estudo, a desigualdade decresce aproximadamente em 0,19\%. Assim sendo, é fundamental a orientação e formulação de políticas públicas para redução da desigualdade com enfoque na educação.

A segunda contribuição mais importante na explicação da desigualdade é da variável Produto Interno Bruto. Verifica-se que para cada aumento de $1 \%$ dessa variável a desigualdade decresce em $0,04 \%$. Esses resultados corroboram (com) a literatura, como em Panizza (2002), que, utilizando dados dos estados norte-americanos durante o período de 1940-1980, concluiu que existe uma relação inversa entre desigualdade de renda e crescimento econômico. Em relação à renda do trabalho, o modelo MMG-sistema apresentou impactos positivos na desigualdade. Destaca-se que essa variável não apresentou o sinal esperado economicamente.

A variável impostos arrecadados apresentou sinal positivo e significativo, contribuindo para aumentar a desigualdade de renda no Brasil. Observase que para cada aumento de $1 \%$ na arrecadação, a desigualdade eleva-se em aproximadamente $0,02 \%$. Em assim sendo, a redução de impostos, por meio de política fiscal eficiente, contribuiu na redução da desigualdade de rendimentos no Brasil.

\section{REFERÊNCIAS}

AGHION, P.; BOLTON, P. Distribution and growth in models of imperfect capital markets. European Economic Review, [S. I.], v. 36, p. 603-611, 1992.

AHN, S. C., SCHIMDT, P. Efficient estimation of models for dynamic panel data. Journal of Econometrics, [S. I.], v. 68, p. 5-28, 1995.

ARELLANO, M.: BOND, S. Some tests of specification for panel data: Monte Carlo evidence and na application to employment equations. The Review of Economic Studies, Stockholm, Sweden, v. 58, n. 2, p 277-297, 1991.

; BOVER, O. Another look at the instrumental-variable estimation of errorcomponents model. Journal of Econometrics, [S. I.], v. 68, p. 29-52, 1995.

AZEVEDO, J.P.; FOGUEL, M.N. Uma decomposição da desigualdade de rendimentos do trabalho no Brasil: 1995-2005. In: PAES DE BARROS, R.; FOGUEL, M.N.; ULYSSEA, G. (Eds). Desigualdade de renda no Brasil: uma análise da queda recente. Brasília, DF: IPEA, 2007. v. II, cap. 27. p. 343-364.

BARRETO, F. A. F. D.; JORGE NETO, P. de M.; TEBALDI, E. Desigualdade de renda e crescimento econômico no Nordeste Brasileiro. Revista Econômica do Nordeste, Fortaleza, v. 32, n. esp., p. 842-859, nov. 2001.

BARROS, P. R.; FRANCO, S.; MENDONÇA, R. Discriminação e Segmentação no Mercado de Trabalho e Desigualdade de Renda no Brasil. In: Paes de Barros, R.; Foguel, M. N.; Ulyssea, G. (Eds). Desigualdade de Renda no Brasil: uma análise da queda recente. Brasília, DF: IPEA, 2007. v. II, cap.28. p. 371-400.

BLUNDELL, R.; BOND, S. Initial conditions and moment restrictions in dynamic panel data models. Journal of Econometrics, [S. I.], v. 87, p. 115143, 1998. 
BRASIL. Ministério do Desenvolvimento Social e Combate à Fome. Bolsa Família. Brasília, DF, [2008]. Disponível em:<http://www.mds.gov.br/bolsafamilia/ menu_superior>. Acesso em: 3 out. 2008.

CONDE, K. Pobres pagam mais impostos que os ricos no Brasil. VilaMulher, São Paulo, 2008. Dinheiro - Finanças. Disponível em:<http:// vilasucesso.vilamulher.com.br/materia/financas/>. Acesso em: 3 out. 2008.

ENDERS, W.; HOOVER, G. A. The effect of robust growth on poverty: a nonlinear analisis. Applied Economics, [S. I.], v. 35, p. 1063-1071, 2003.

ENRENBERG, R.G; SMITH, R.S. A moderna economia do trabalho: teoria e política pública. São Paulo: Makron Books, 2000. p 319-409.

HOFFMANN, R. Transferência de renda e a redução da desigualdade no Brasil e cinco regiões entre 1997 e 2004. Econômica, Rio de Janeiro, v. 8, n. 1, p. 55-81, jun. 2006. Disponível em: <http://www.uff.br/cpgeconomia/ economica.htm>. Acesso em: 3 out. 2008.

KAKWANI, N.; NERI, M.; SON, H. Linkages between pro-poor growth, social programmes and labour market: the recent Brazilian experience. Working Paper, Brasília, DF, n. 26, p. 1-39, aug. 2006.

KUZNETS, C.G. Economic growth and income inequality. The American Economic Review, Pittsburgh, PA, v. 45, n. 1, p. 1-28, mar. 1955.

MARINHO, E.; LINHARES, F; CÂMPELO, G. Os programas de transferências de renda do governo impactam a pobreza no Brasil? Fortaleza: UFC/CAEN, 2007. (Série Ensaios sobre a pobreza, n. 12). Disponível em:<http://www.caen.ufc.br/lep. htm>. Acesso em: 3 out. 2008.

PANIZZA, U. Income inequality and economic growth: evidence from American data. Journal of Economic Growth, [S. I.], v. 7, n. 1, p. 25-41, mar. 2002.

ROCHA, S. Pobreza no Brasil. Afinal de que se trata? 3. ed. Rio de Janeiro: Editora FGV, 2006.

SCHULTZ, T. W. Capital formation by education. Jornal of political economy, Chicago, v. 68, n. 6, p. 571-583, dec. 1960.

Investiment in human capital. The American Economic Review, Pittsburgh, PA, v. 51, n. 68, p. 17-68, 1961.

SILVEIRA, F.G. Impactos das transferências governamentais e da tributação na distribuição de renda no Brasil: considerações sobre o documento gasto social do governo central - 2001 e 2002, da secretaria de política econômica do ministério da fazenda. Rio de Janeiro: IPEA, 2004. (Ensaios políticas sociais - acompanhamento e análise, n. 1).

VIANA, S. W. etal. Tributação e desigualdade social no Brasil. Rio de Janeiro: IPEA, 2001. Disponível em:<http://www.fase.org.br/projetos/vitrine/admin/ Upload/1/File/Proposta88_89/werneck_vainna_e_ outros.PDF>. Acesso em: 3 nov. 2008.

WINDMEIJER, F. A finite sample correction for the variance of linear efficient two-step GMM estimators. Journal of Econometrics, [S. I.], v.126, p.25-51, 2005.

\section{NOTAS}

1 A PNAD não foi realizada no ano de 2000. Em assim sendo, calculou-se as médias aritméticas das variáveis dos anos de 1999 e 2001 para compor os dados do ano de 2000

2 Os estados da região Norte não foram considerados na amostra em função da não disponibilidade de dados para a zona rural antes de 2004.

\section{Jair Araújo}

Engenheiro de Pesca

Doutor em Economia pela Universidade Federal do Ceará (UFC)

Professor do Curso de Pós-Graduação em Economia Rural da Universidade Federal do Ceará (UFC)

E-mail: jairandrade@ufc.br

\section{Emerson Marinho}

\section{Estatístico}

Doutor em Economia pela Fundação Getulio Vargas (FGV) Professor do Curso de Pós-Graduação em Economia -Universidade Federal do Ceará (UFC)

E-mail: emarinho@ufc.br

Universidade Federal do Ceará - UFC Avenida Mister Hull, S/N - Pici, Fortaleza - CE CEP: $60455-760$ 